\title{
Procesos socio-ambientales de tecnología e innovación
}

\author{
De las brujas alquimistas de ayer \\ a las científicas ambientales de hoy \\ Social-environmental processes of technology and innovation. \\ From the alchemist witches of yesterday to the \\ environmental scientists of today
}

\author{
María Eugenia Castro Ramírez*
}

Citar este artículo como: Castro Ramírez, M. E. (2018). Procesos socio-ambientales de tecnología e innovación. De las brujas alquimistas de ayer a las científicas ambientales de hoy. Revista Nodo, 13(25), pp. 34-43

\section{Resumen}

Los procesos socio-ambientales de tecnología e innovación, son en este trabajo, una alternativa de abordaje científico, resultado de una mirada crítica a la manera de pensar y hacer ciencia, desde una ciencia dominante, masculina y de élites, construida a través de siglos, excluyendo a las mujeres, a sus formas de ser, conocer y aprehender el mundo y en él, el mundo de las ciencias, en plural. Muestra, así mismo, como rompiendo esos paradigmas, un grupo de investigadoras, reconstruye la línea de las mujeres en la producción científica y lo hace con sus compañeros hombres, en una nueva forma de hacer ciencia, más igualitaria, plural y diversa, explicada a través del proceso de formación de su área de investigación, así como con los resultados de su producción científica.

\section{Palabras clave}

Medio ambiente, Mujeres y ciencia, Tecnología e innovación.

\section{Abstract}

The social-environmental processes of technology and innovation that are in this work, are an alternative scientific approach, the result of a critical look at the way of thinking and doing science, from a dominant, masculine and elite science, built through centuries, excluding women and their ways of being, knowing, and understanding the world, the environment, and the world of sciences, in the plural. It shows, as well as the breaking of these paradigms, a group of researchers that reconstructs the line of women in scientific production and does it with their fellow men, in a new way of doing science, more egalitarian, plural and diverse, explained through the formative process of its area of research, as well as the results of their scientific production.

\section{Keywords}

Environmental science, Women and science, Technology and inovation.

Fecha de recibo: 05 de julio de 2018 - Fecha de aceptación: 07 de diciembre de 2018

* Profesora-investigadora. Universidad Autónoma Metropolitana Unidad Xochimilco, México. Correo electrónico: eugeniacastro@me.com 


\section{Introducción}

Este trabajo tiene dos partes. Una que desarrolla el camino seguido por la ciencia, hasta que se consolida como ciencia dominante, donde se excluye la línea de construcción de la ciencia iniciada por las mujeres en el medioevo, hasta el día de hoy. La otra muestra el camino seguido por un grupo de investigadoras e investigadores que buscan romper ese paradigma existente y recuperar la visión femenina en el quehacer científico, desde una nueva epistemología y metodología más igualitaria, porque además de incluir la perspectiva de género, produce innovación científica y tecnológica, de manera interdisciplinaria, donde se reivindican las distintas miradas, métodos, técnicas e instrumentos, desde lo que es el corazónn de la División de CyAD, las Ciencias y Artes para el Diseño, dualidad que enriquece el proceso creativo de las ciencias, en plural.

Cuestiona, pero propone e innova, construyendo conocimiento a lo largo del proceso científico.

\section{Fundamento y justificación del} tema

El tema tiene su origen, de un lado, en el proyecto interdisciplinario de investigación Modelos socioambientales para evaluar el Cambio Climático y prevenir riesgos en México, y por el otro, derivado de mi participación en el Comité multidisciplinario y organizador del $\mathrm{V}$ Congreso Internacional de Avances de las Mujeres en las Ciencias, las humanidades y todas las disciplinas: Globalización y tecnologías de vanguardia. Del vínculo de los dos, se deriva este trabajo.

Pertenecer a la red TAI de la UAM Xochimilco y haber sido invitada a participar con una conferencia magistral en el $2^{\circ}$ Simposio Internacional de Tecnología, Ambiente e Innovación, me brinda la oportunidad de mostrar, con una visión de género y crítica, los avances de las mujeres en las ciencias, y en las ambientales particularmente y su articulación con las funciones sustantivas de la UAM, la investigación, docencia, preservación y difusión de la cultura, y el servicio universitario.
En la División de Ciencias y Artes para el Diseño, es relativamente reciente, desde la creación de los programas de maestría y Doctorado en Ciencias y Artes para el Diseño en 1996, la investigación interdisciplinaria y transdisciplinaria, la cual viene a tener un papel relevante en la producción de conocimientos, soportada en la complejidad de los problemas emergentes, tales como los del deterioro del medio ambiente y por ende de la calidad de vida de los habitantes del planeta, problemas que implican ser abordados desde diferentes disciplinas. Y no solo de ellas, sino además desde una nueva manera de construir el conocimiento, desde lo femenino y lo masculino, que rompa con los paradigmas hasta ahora existente y vuelva la mirada hacia la innovación y nuevas tecnologías, en el marco de la sustentabilidad y habitabilidad, en nuestro caso.

\section{Marco epistémico y metodológico}

Los métodos tradicionales de ser y hacer ciencia, son ahora puestos en cuestión y dan cabida, no sin resistencias, a una visión holística, así como a las teorías de la complejidad, de la sistémica y de la cibernética, con sus propias metodologías.

La ciencia dura y los métodos científicos tradicionales, dan paso a métodos usados en las ciencias sociales y humanas, como los etnográficos hasta los que hoy llamamos métodos cualitativos.

Lo anterior implica volver la mirada hacia la antropología, pero a su vez, hacia la ecología y así van convergiendo las distintas ciencias; simultáneamente emergen ante los ojos de los científicos, investigadores y académicos, los "otros conocimientos" de "otras" y "otros", de los pueblos originarios, de las luchas de las mujeres, de los ecologistas, de los inmigrantes, de los desplazados, de las minorías, entre otros. Esto da lugar a otra ciencia, de eso se trata este artículo.

Como es imposible cubrir todos los campos de la ciencia actual y dada la complejidad que ello implica, me limitaré en este artículo a mostrar 
como lo hemos realizado un grupo de mujeres y hombres que lucharon muchos años para que se les reconociera como área de investigación y que cuando no estaba de moda, como hoy se le llama el tema ambiental, supieron mantener o resistir por más de 20 años, las y los mayores, y menos tiempo, pero con el mismo valor, los jóvenes investigadores que formamos en el posgrado y en el Área Espacios Habitables y Medio Ambiente. Llamada así, porque articula e interrelaciona dos ejes, los de la sustentabilidad y la habitabilidad, en una hipótesis que seguimos sosteniendo, porque los nuevos conocimientos y el tiempo nos han dado la razón, sin la primera es imposible la segunda.

Los métodos usados son de tipo cuali-cuantitativo, la investigación de acción participativa o de recorrido sensible, por señalar algunos, y como herramientas de interpretación fueron implementados programas (software) como el Modelo Sensible de Vester o el Análisis de Ciclo de Vida, según fuera adecuado para obtener la mejor información y cumplir alcances y objetivos. Así mismo, utilizamos índices e indicadores y métricas de validación; sistemas de monitoreo y prospección.

Para concluir esta parte diré que las mujeres de este grupo, hemos tenido como investigadoras y científicas, si dicho así sin miedo, que sortear la misma lucha que nuestras compañeras en otros ámbitos, con la diferencia de tener unos compañeros que nos cobijan, para juntos cumplir con nuestros sueños de hacer lo que más nos gusta, ser los mejores investigadores y docentes, para esta y futuras generaciones y contribuir a dilucidar los problemas de nuestro tiempo y generar propuestas de solución viables para las mayorías del país y del planeta, las y los desposeídos, y para preservar nuestra casa común, la tierra. Reiterando con esto que no es cierto que la ciencia sea neutra.

A propósito del nombre de este artículo, quise desafiar la noción convencional y equívoca de pretender que la ciencia es masculina y que se origina en sólo la razón. El conocimiento y las formas de adquirirlo están mediados históricamente por las cosmovisiones y las formas de percibirnos y percibir el mundo y el universo, de aprehenderlo y aprenderlo, de relacionarnos con él. Lo hacemos de diferentes maneras, desde el género al que pertenecemos.

No me voy a remontar al momento en que evolucionamos hasta ser homínidos, pero si quiero traer la historia de "Lucy" en Sterfontein en Sudáfrica1, aquella pequeña australopiteca que en tránsito a la evolución humana se convirtió en nuestro ancestro directo (Clarke \& Tobias, 1995), según la última datación, hace un poco más de tres y medio millones de años (Granger et al., 2015), pero que conservó sus "instintos", diría yo su esencia femenina, pasando por las "brujas alquimistas" del medioevo, hasta las científicas de hoy, con aportes críticos a la ciencia (Blazquez, 2011).

\section{Las brujas alquimistas}

Me detengo en el libro de la autora antes nombrada, El retorno de las brujas, porque nos muestra, de un lado, que el hecho de que las mujeres conservaran un pensamiento mágico, que fueran parteras, curanderas, yerberas y realizaran ceremonias de la fertilidad, para favorecer la agricultura, así como prácticas de adivinación entre otras cosas, las hacía creadoras de conocimiento empírico, es decir poderosas, en una época de la historia (s. XIV al XVII) de cambios sociales, culturales, económicos y poblacionales centrados en la secularización y en el control de las élites, entre ellas el gobierno y la iglesia, quienes detentaban el poder, y al que no podían desafiar, las clases populares y su cultura, a través de lo que ellos llamaron la magia baja o brujería. Aunque cabe señalar que la naciente ciencia de las élites dominantes se derivaba también de la magia alta o culta como la astrología, la alquimia y la nigromancia era practicadas por sectores de la élite como el clero y los médicos. La magia del pueblo, fue estigmatizada y perseguida, la de las élites no. (Blazquez, 2011). Ese es el origen de la ciencia.

1 Sitio localizado a pocos kilómetros de Johannesburgo, la capital de Suráfrica y declarado por la UNESCO, Cuna de la humanidad. 
De otro lado, está lo que Blazquez denomina "La persecución de brujas desde la perspectiva de género" (2011, p. 23), en donde demuestra que la mayoría de los procesos por "brujería" seguidos en Europa, hasta en 95\% en algunos lugares, fue contra mujeres, a quienes la cultura dominante, negaba el derecho al conocimiento y menos al que no fuera del dominio masculino. Contradictoriamente consideraban a las mujeres seres subalternos, dependientes y frágiles.

Sostiene la autora que "Si bien la creencia popular en brujas y hechiceras capaces de dañar existía desde el mundo grecolatino, no es sino hasta el siglo XIV cuando teólogos, clérigos y hombres dedicados a la ciencia aceptan la creencia, la difunden y desarrollan un sistema de normas y códigos para abordar los actos de brujería en los llamados tratados demonológicos" (Blazquez, 2011, pp. 23-24).

Cabe destacar que los conocimientos que desarrollaron las llamadas brujas, fueron obtenidos con métodos e instrumentos, que la ciencia de hoy reconocería: su sustento empírico, la observación de los fenómenos astrológicos y climáticos, de la naturaleza misma, al estudiarla, clasificarla y determinar sus propiedades. Así como su aplicación práctica a los oficios que ejercían, en los rituales y ceremonias religiosas, en la perfumería, la medicina, la agricultura, entre otras.

En cuanto a las técnicas, sostiene Blazquez que “... desarrollaron las técnicas químicas de la destilación, la extracción y la sublimación utilizadas por los alquimistas." (2011, p. 26)

Las cocinas eran sus "laboratorios" y crearon artefactos apropiados como alambiques, donde trataban los metales, por ejemplo.

No cabe duda que esas mujeres-brujas, construyeron conocimientos para servir al pueblo, del que no se ocupaban las élites cultas y a las que desafiaban. Otro tipo y forma de conocimiento diferente al dominante, otra cultura que combinaba el manejo de la alquimia y el ritual, a decir de Blazquez “... en los procesos de brujería no solo se perseguía a la magia o a las mujeres sino a la magia de las mujeres, y que una de las principales razones para perseguirlas era la intolerancia a los conocimientos relacionados con la sexualidad y la vida que dominaban y practicaban desde épocas ancestrales, y que era necesario controlar" (2011, p. 30).

Es en este periodo de la edad media donde se produce la cacería de brujas, como ya vimos, en el cual también emerge la ciencia y se extiende al renacimiento y al Siglo XVII, época donde se construye la ciencia moderna occidental y se destruye simultáneamente “... una línea de conocimiento, la de las mujeres..." (Blazquez, 2011, p. 32).

Posteriormente, durante siglos, ya no aparecen las mujeres en el campo de las ciencias, hasta principios del Siglo XIX, que lo hacen en un ámbito doméstico y a finales del siglo, institucionalmente, de manera incipiente en las universidades. No me voy a ocupar más de este periodo, de los que hay numerosos estudios, sino sólo para señalar que la incorporación se hace a partir de una ciencia de hombres para hombres, pero de manera progresiva las mujeres van conquistando espacios, no solo como estudiantes universitarias, sino posteriormente como docentes y finalmente como investigadoras (mediados del Siglo XX y lo transcurrido del XXI).

Lo anterior viene a modificar a las instituciones mismas, a los campos a los que se dedicaban las mujeres o les daban acceso en un principio, y hoy abriéndose camino a espacios vedados, considerados masculinos, como los de las llamadas ciencias duras, las ingenierías y por supuesto los de la innovación y la tecnología, en los que hoy ya incursionan.

\section{Las mujeres científicas de hoy}

Aún hoy, la ciencia conserva la mirada masculina, para lo que me remito a los datos duros del Conacyt, para nuestro país, donde las mujeres 
investigadoras no sólo siguen siendo minoría, sino que son evaluadas por los miembros del Sistema Nacional de Investigadores de nivel III, que son mayoritariamente hombres y en sus evaluaciones, impiden que las mujeres entren al Sistema o escalen dentro de él a niveles superiores. (Conacyt, 2015)

Los datos a nivel nacional se corroboran con los de nivel mundial, donde si bien las mujeres hoy son $50 \%$ del total de la matrícula a nivel universitario y más o menos el 30\% de investigadoras del total, estos números bajan cuando se trata de Ingenierías y tecnología, donde representan el 15\% y menos en cargos de dirección de empresas, donde los procesos de innovación y tecnología son centrales.

Las decisiones sobre qué ciencia y tecnología necesitamos como humanidad y país, está fuera del poder de decisión de las mujeres, ya que la representación es mínima, aún con las cuotas de género, en el Consejo Nacional de Ciencia y Tecnología (CONACYT), en el Congreso de la Unión, etc.

Estamos en la producción científica construida por los varones, con su mirada, sus teorías, sus métodos e instrumentos. Sin embargo, habría que destacar que hay pequeños nichos subalternos, donde se empieza a reconstruir esa otra línea de la ciencia que se perdió en el medioevo, la de las mujeres, con otras miradas, otros caminos, otras técnicas.

\section{Esbozo de un ejemplo de aplicación Interdisciplinaria}

La construcción del área de investigación, Espacios Habitables y Medio ambiente y los tres proyectos colectivos de investigación que hemos realizado durante nuestra existencia: Ciudad y medio ambiente, Desarrollo regional, recursos y diseño ambiental en la cuenca del Papaloapan y Modelos socio-ambientales para evaluar el Cambio Climático y prevenir riesgos en México ${ }^{2}$, son un buen ejemplo de aplicación interdisciplinaria y cientí-

2 Este último, realizándose actualmente. fica, de procesos socio-ambientales de tecnología e innovación, pero también de equidad de género, en los procesos investigativos del grupo de investigadores.

Siendo coherentes con el planteamiento teórico metodológico de este artículo, resulta necesario explicar, que esa visión de género permeó la construcción del proyecto de creación de nuestra Área, que inserta en una División como la de CyAD y en un departamento llamado de Métodos y Sistemas, se constituyó en una novedad, por que reivindicaba el trabajo colectivo, cooperativo, no individual de investigación, que era el que se hacía en la División, cuando la había, y donde en la Universidad todavía no se hablaba de la necesidad de realizar proyectos colectivos de investigación, a pesar de la organización del trabajo de tipo departamental y el sistema modular propio de la UAM Xochimilco, que así lo postulaba.

También resultó una novedad que participaran en el grupo, investigadoras e investigadores de tres departamentos de la División, provenientes de disciplinas diferentes e invitados de otras universidades. Nuestros orígenes eran diversos, lo que era común, es que estábamos decididos a hacer investigación científica y todas y todos teníamos diferentes grados de avances en nuestra carrera como investigadoras e investigadores y nos estábamos habilitando con estudios de maestría y doctorado.

Nos propusimos construir un lenguaje común, para entendernos, dado que todos proveníamos de disciplinas distintas, geografía, matemáticas, economía, arquitectura y urbanismo.

En ese momento, dos problemas emergentes y relevante, los procesos de urbanización acelerada de las ciudades latinoamericanas y el deterioro ambiental, nos unieron en torno al primer proyecto de investigación que abordamos, el de Ciudad y medio ambiente. De ahí pasamos a construir una epistemología ambiental. Esto a partir de un seminario permanente, donde leíamos a los autores más destacados en el tema, propuestos por cada uno de las y los integrantes (Figura 1). 


\section{EPISTEMOLOGÍA AMBIENTAL}

\author{
EL SIGLO DE LA TIERRA \\ S. XXI \\ ECOCENTRISMO \\ HOLISMO \\ MARXISMO EDOLÓGICO \\ SUSTENTABILIDAD \\ BIOGEOGRAFÍA \\ ECOLOGÍA HISTORIA AMBIENTAL
}

Figura 1. Paquete didáctico (manual), Epistemología Ambiental. Fuente: elaboración propia (2015)

En esa etapa, de manera simultánea desarrollamos la investigación colectiva, todavía de carácter multidisciplinario, y publicábamos de forma individual los resultados. Esto hasta 2001, donde ya habíamos construido además de un lenguaje común, un marco epistémico sólido y una metodología ambiental común (figura 2).

\section{METODOLOGÍA AMBIENTAL}

\section{MÉTODOS CUALI-CUANTITATIVOS}

\author{
SISTEMAS COMPLEJOS \\ BIOCIBERNÉTICA \\ INVESTIGACIÓN-ACCIÓN PARTICIPATIVA \\ ECODISEÑO \\ PERMACULTURA \\ DISEÑO AMBIENTAL
}

Figura 2. Paquete didáctico (manual) Metodología ambiental. Fuente: elaboración propia (2017)

Para ese momento ya habíamos incorporado al Seminario del grupo de investigación a alumnas y alumnos de maestría y doctorado en Cyad, que con sus propios trabajos de investigación se articulaban orgánicamente al grupo de trabajo de investigadores consolidados y abonaba a los procesos de enseñanza-aprendizaje de las Ciencias y Artes para el Diseño, particularmente en las tres áreas de posgrado en las que participábamos como docentes y tutores: Sustentabilidad Ambiental, Investigación y Gestión Territorial e Innovación Científica y Tecnológica.

Así mismo los alumnos del Área de Sustentabilidad de la Maestría en CyAD publicaban con nosotros sus primeros artículos de investigación y los de licenciatura de nuestros grupos de docencia en Arquitectura, participaban de talleres teóricoprácticos, donde se vinculaba lo ambiental con la innovación tecnológica y se publicaban los resultados (figura 3).

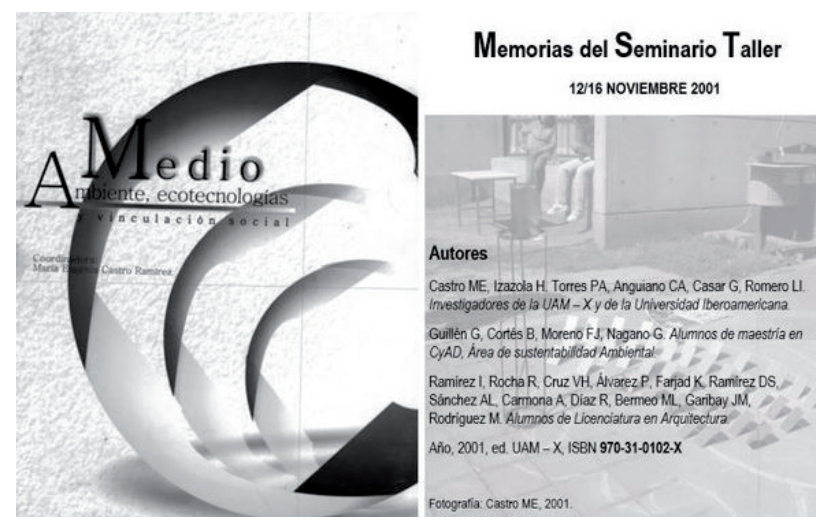

Figura 3. Libro Medio Ambiente, ecotecnologías. Vinculación social. Fuente: Castro (2001).

La postura epistemológica del grupo fue publicada en un artículo que arrojó resultados colectivos de investigación (Castro et al., 2001).

Desde la conformación del grupo, planteamos la necesidad de vincular, investigación, docencia, preservación y difusión de la cultura, y servicio universitario, lo que ha sido una constante hasta hoy.

Para 2006, se publicó el libro colectivo, Los problemas de los espacios habitados y el medio ambiente, donde participamos como autores: María Eugenia Castro y Paola Martínez, Laura Isabel Romero, María del Carmen Ramírez, Manuel Lerín, y Juan José Zoreda. Este mostraba los resultados del primer proyecto de investi- 
gación colectiva, Ciudad y medio ambiente y se daban los pasos para integrar dos ejes de investigación los de los espacios habitados y el medio ambiente (Castro y Romero, 2006).

Antecedente del documento de creación del área de investigación Espacios Habitables y Medio Ambiente.

El segundo proyecto de investigación colectivo, Desarrollo regional, recursos y diseño ambiental en la Cuenca del Papaloapan es el resultado del financiamiento de la primera convocatoria del rector general de la Universidad Autónoma Metropolitana (UAM), Dr. José Lema Labadie para financiar proyectos multidisciplinarios en el que participaran grupos de investigación consolidados de al menos dos Unidades de la Universidad. El Área nuestra participó con dos proyectos, uno con investigadores de la UAM Cuajimalpa y Azcapotzalco y otro con investigadores de la UAM Iztapalapa. Este último fue el que ganó, con otros cuatro más de toda la Universidad.

El Área ya había establecido vínculos con esos otros grupos de investigación y eso nos permitió hacerlo formalmente con los de Iztapalapa, formado por antropólogos, economistas e ingenieros ambientales. Diferentes enfoques y problemas de diversa índole hicieron que el trabajo que se llevó a cabo en una primera etapa de manera armoniosa, a través de seminarios, trabajo de campo e interrelaciones con las comunidades de la zona de estudio, al final terminó funcionando como dos equipos, el de la UAM Xochimilco y la UAM Iztapalapa. A pesar de las dificultados que eso entrañaba, entregamos los informes correspondientes y los productos a los que nos habíamos comprometido y en este artículo asentamos la dificultad e imposibilidad de la integración pretendida con el proyecto.

Lo destacable, es que, al obtener recursos suficientes para un proyecto de largo alcance (cinco años), nos permitió, adquirir los instrumentos necesarios, para llevar a buen término la investigación.
En ese lapso (2007-2011), nos consolidamos internamente como equipo de investigación y de manera relevante, construimos una plataforma metodológica apropiada, donde lo femenino y lo masculino se pudieran expresar y nos permitiera abordar investigaciones interdisciplinarias, en el ámbito del diseño ambiental, junto con herramientas con un alto grado de innovación, como el software de Vester (figura 4).

\section{INSTRUMENTOS METODOLÓGICOS DE LA CIENCIA AMBIENTAL (Software) \\ MOdELO SENSIBLE DE VESTER \\ ANÁLISIS DE CICLO DE VIDA \\ ANÁLISIS DE IMPACTO AMBIENTAL HUELLA DE CARBONO \\ ANÁLISIS DE RIESGO Y VULNERABILIDAD \\ ESCENARIOS DE CAMBIO CLIMÁTICO}

Figura 4. Paquete didáctico (Manual). Instrumentos Metodológicos de la Ciencia Ambiental (Software). Fuente: elaboración propia

Para llevar a cabo la investigación utilizamos como herramienta de interpretación (análisis), diversos métodos cualitativos, como el permacultural, la Investigación Acción Participativa, el Recorrido Sensible y otros de tipo cuantitativo como el Análisis Estadístico, y la Modelación Matemática, entre otros, pero todos dentro del modelo Sensible (sensitivo o de sensibilidad) de Vester, de tipo cuali-cuantitativo, considerado por su recursividad también como biocibernético (figuras 5 y 6).

Los resultados de esta segunda investigación colectiva e interdisciplinar, se muestran en dos etapas: la primera con la publicación del libro Diseño Ambiental en la Cuenca del Papaloapan de María Eugenia Castro, Alberto Cedeño, Manuel Lerín, Laura Isabel Romero y Pablo Torres (Castro et al., 2009) (Figura 7). 
Estructura recursiva del Modelo Sensible*

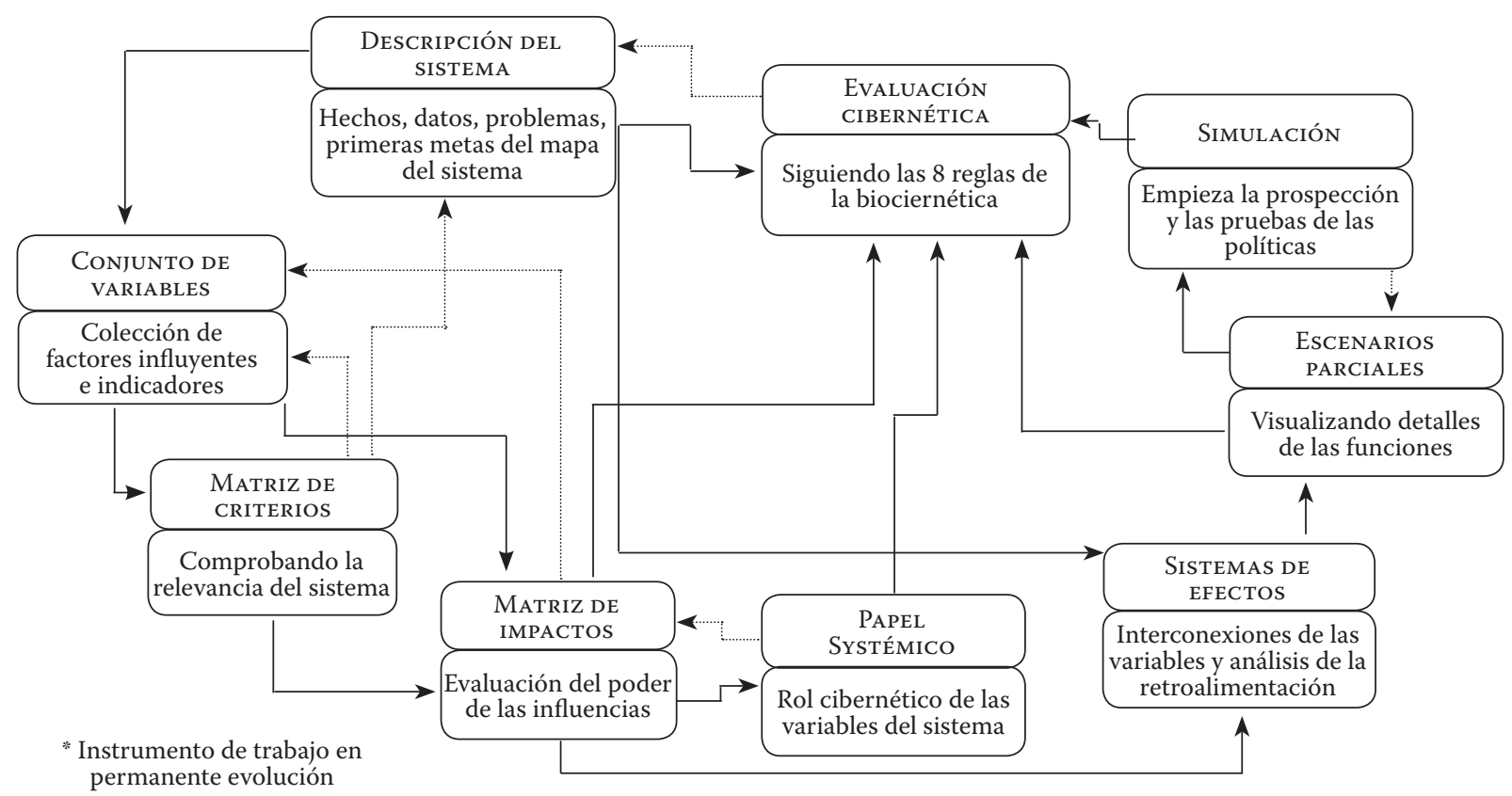

Figura 5. Manual del modelo de sensibilidad. Estructura recursiva del Modelo Sensible*. Fuente: Vester (2008, p. 15)

\begin{tabular}{|c|c|c|c|}
\hline Método & Pasos del sistema & Actividades & Productos \\
\hline $\begin{array}{l}\text { Modelización del sistema } \\
\text { Desarrollo Regional, } \\
\text { recursos y diseño ambiental } \\
\text { en la Cuenca del Papaloapan } \\
\text { Método cuali-cuantitativo } \\
\text { Participativo: Modelo sensible } \\
\text { (MS) } \\
\text { Actores: Pobladores, Investi- } \\
\text { gadores y Autoridades } \\
\text { Responsable: María Eugenia } \\
\text { Castro Ramírez } \\
\text { Investigadores: Salvador } \\
\text { Duarte, Manuel Lerín, María } \\
\text { del Carmen Ramírez, Laura } \\
\text { Romero y María Eugenia } \\
\text { Castro } \\
\text { Investigadores invitados: } \\
\text { Primera etapa: Alberto } \\
\text { Cedeño y Pablo Torres } \\
\text { Segunda etapa: Herman } \\
\text { Barrera, María Alejandra } \\
\text { Hernández y José Luis Gutié- } \\
\text { rrez }\end{array}$ & $\begin{array}{l}\text { - Descripción del sistema } \\
\text { - Conjunto de variables } \\
\text { - Matriz de criterios } \\
\text { - Matriz de impactos } \\
\text { - Rol sistemático } \\
\text { - Sistema de efectos } \\
\text { - Escenarios parciales } \\
\text { - Simulación } \\
\text { - Evaluación cibernética }\end{array}$ & $\begin{array}{l}\text { Hechos, datos, problemas y } \\
\text { metas } \\
\text { Recorrido sensible y entrevistas } \\
\text { Colección de factores de } \\
\text { influencia e indicadores } \\
\text { Sistematización de la informa- } \\
\text { ción } \\
\text { Comprobando la pertinencia del } \\
\text { sistema } \\
\text { Participación de actores } \\
\text { Evaluación de la validez de los } \\
\text { impactos } \\
\text { Participación de todos los } \\
\text { actores } \\
\text { Papel cibernético de variables en } \\
\text { el sistema } \\
\text { Carga de datos al programa MS } \\
\text { Eslabones o enlaces de las } \\
\text { variables y análisis de retroali- } \\
\text { mentación (feedback) } \\
\text { Aplicación y resultados en el MS } \\
\text { Pronósticos y prueba del sistema } \\
\text { Aplicación y resultados en el MS } \\
\text { Participación todos los actores } \\
\text { Evaluación del sistema siguiendo } \\
\text { los pasos del biocibérnetica MS }\end{array}$ & $\begin{array}{l}\text { Primer mapa del sistema } \\
\text { Tabla concentrada de recu- } \\
\text { rrencias y tablas del sistema de } \\
\text { variables y definiciones } \\
\text { Tabla de Matriz de criterios y } \\
\text { variables } \\
\text { Tabla con Matriz de impactos } \\
\text { por actores y consensuada } \\
\text { Gráfica del Sistema de roles de } \\
\text { variables de relaciones de } \\
\text { Gráficas de gráficas } \\
\text { efectos del sistema y gerco- } \\
\text { de las funciones de intercona } \\
\text { nexión positiva y negativa } \\
\text { Gráficas de escenarios } \\
\text { parciales (EP) } \\
\text { Gráficas de simulación líneal } \\
\text { de las tendencias de los EP } \\
\text { Simulación de los escenarios } \\
\text { parciales con políticas o } \\
\text { acciones de intervención } \\
\text { Tabla de avaluación del sistema }\end{array}$ \\
\hline
\end{tabular}

Figura 6. Paquete didáctico (manual) Metodo Biocibernético. Modelo Sensible de Vester. Fuente: elaboración propia, 2017 


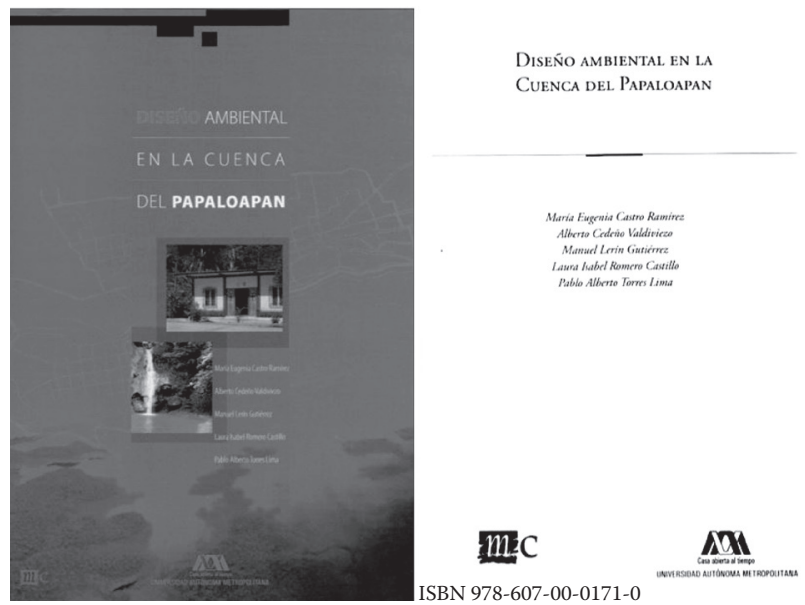

Figura 7. Libro. Diseño Ambiental en la Cuenca del Papaloapan, 2017. Fuente: propia

Una segunda donde los resultados finales de la investigación se presentan en el libro colectivo Desarrollo ambiental en la Cuenca del Papaloapan, escrito a su vez por María Eugenia Castro, Salvador Duarte, Manuel Lerín, Laura Isabel Romero, María Alejandra Hernández, Herman Barrera y José Luis Gutiérrez ${ }^{3}$ (Castro et al., 2017) (Figura 8).

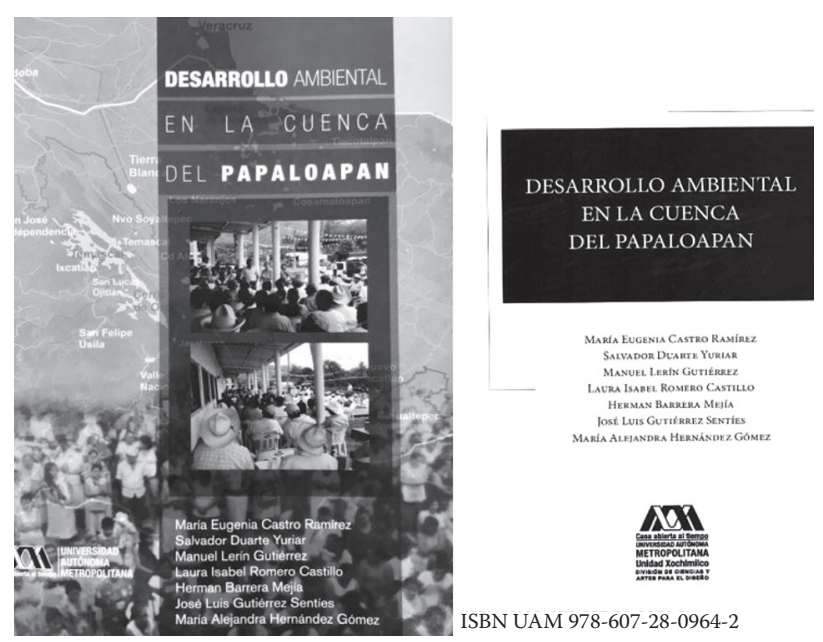

Figura 8. Libro. Desarrollo Ambiental en la Cuenca del Papaloapan, 2018. Fuente: propia

3 Los tres últimos, fueron alumnos del Doctorado en CyAD, en el Área de Sustentabilidad Ambiental y hoy todos tienen el grado de doctorado.

\section{Conclusiones}

Las mujeres somos constructoras de conocimientos desde que existimos en el planeta, las brujas alquimistas y las científicas de hoy así lo demuestran. Si hiciéramos un recorrido por la historia más a profundidad, seguramente encontraríamos otros hallazgos significativos, por ejemplo en etapas más tempranas, anteriores a las familias sindiasmica y punalúa (patriarcales), las mujeres construyen conocimientos soportados en un mundo real y simbólico: el matriarcado, las diosas, el caos como origen femenino del universo, la vida misma.

Si bien no es el objeto de este artículo una visión histórica y menos desde la historia escrita por varones, si será importante, así como hace falta escribir la historia ambiental, también escribir la historia de la ciencia que incluya la ciencia femenina, en todas sus dimensiones.

El origen de este artículo, dictar una conferencia magistral de innovación, ciencia, tecnología y ambiente dirigida a académicos e investigadores varones en su mayoría, me abría una oportunidad para dirigirme a ellos, desde lo que nos une, el conocimiento, pero desde la alteridad (otredad), en la búsqueda de una nueva época de reconocimiento, de conciliación y síntesis de alguien que no se ha considerado feminista, pero que cotidianamente ha vivido en el ámbito académico-científico, uno de los más liberales de nuestra sociedad, el poder patriarcal, ejercido, por que no decirlo por hombres y mujeres. Esperando sea un pequeño aporte a ese cambio tan necesario en el camino de la construcción de la ciencia y la vida cotidiana.

No encontré mejor manera de hacerlo que mostrando dos épocas, mirando el quehacer de la ciencia de las mujeres de la edad media y del trabajo de nuestro equipo de investigación en pleno siglo XXI, cuyo centro es la ciencia ambiental, donde pueden leerse objetivos, métodos, instrumentos, resultados, pero mediados por las subjetividades, la mirada y el sentir de lo femenino que emerge en las dos épocas. 
Si se reconoce que la ciencia es un constructo de conocimientos, entonces habrá que concluir diciendo, que las mujeres -del Área y las alumnas de posgrado que han participado en ella- hacemos ciencia y que para ello estamos utilizando métodos propios, holísticos, suaves, colectivos e instrumentos como la sonrisa, el abrazo, la cercanía, la solidaridad, todos ancestrales, junto con los denominados duros. Hoy se resumen en la epistemología de la complejidad, en los métodos cualitativos, pero también nos hemos apropiado de las tecnologías de la información y de la alta tecnología. Utilizamos software sofisticados y ciencias físicas y matemáticas, pero también hacemos filosofía e historia, por señalar algunas, construimos modelos y hacemos prospectiva. Tenemos contribuciones importantes en la creación de las ciencias de la vida y las ciencias ambientales, todas interdisciplinarias. De esa manera creamos, porque no hay nada más creativo que un proceso de construcción científica. Somos artistas que tejemos la red investigativa.

En tanto no reconozcamos, hombres y mujeres, que razonar y sentir, están en ambos, pero que las formas de dominación de la cultura, nos asignó papeles diferentes, no habremos de evolucionar hacia formas más equitativas y complementarias, que se verán reflejadas, también en la construcción de las ciencias, en plural.

\section{Referencias bibliográficas}

Blazquez, N. (2011). El retorno de las brujas. Incorporación, aportaciones y críticas de las mujeres a la ciencia, México: UNAM.

Clarke, R y Tobias, P. (1995). Sterkfontein member 2 foot bones of the oldest South African hominid, Science, (269), pp. 521-524.

Granger D.E. et al. (2015). New cosmogenic burial ages for Sterkfontein Member 2 Australopithecus and Member 5 Oldowan, Nature, Vol. 522, pp. 85-88, publicado online el 01 de abril de 2015.

Castro, M.E. y otros. (2001). Habitabilidad, medio ambiente y ciudad en la revista Ciudades, No. 51, pp. 10-18. Puebla, México: RNIU

Castro, M.E. coord. (2002). Medio ambiente, ecotecnologías y vinculación social, México: UAM Xochimilco.

Castro, M.E. y Romero L.I. (2009). Los problemas de los espacios habitados y el medio ambiente, UAM Xochimilco, México.

Castro, M.E., Cedeño, A., Lerín, M, Romero, L.I. y Torres, P. (2009). Diseño ambiental en la Cuenca del Papaloapan. México: Mc -UAM.

Castro, M.E., Duarte, S., Lerín, M., Romero, L.I., Hernández, M.A., Barrera, H. y Gutiérrez, J.L. (2017). Desarrollo ambiental en la cuenca del Papaloapan. México: Colofón-UAM/X.

CONACYT (2015). CTIndicadores en Ciencia, tecnología e innovación, México: ConacytINEGI- FCCyT-SE-SEP.

Vester, F. (2008). Manual del modelo de sensitividad Prof. Vester, St. Gallen AG: Malik Management Zentrum. 